\title{
Significance of Multimodality Therapy in Patients with a Superior Sulcus Tumor of the Lung: A Review Article
}

\author{
Gouji Toyokawa, Mitsuhiro Takenoyama, Yukito Ichinose
}

Department of Thoracic Oncology, National Kyushu Cancer Center, Fukuoka, Japan.

Email: yichinos@nk-cc.go.jp

Received December 20 ${ }^{\text {th }}, 2013$; revised January $10^{\text {th }}, 2014$; accepted January $17^{\text {th }}, 2014$

Copyright (C) 2014 Gouji Toyokawa et al. This is an open access article distributed under the Creative Commons Attribution License, which permits unrestricted use, distribution, and reproduction in any medium, provided the original work is properly cited. In accordance of the Creative Commons Attribution License all Copyrights (c) 2014 are reserved for SCIRP and the owner of the intellectual property Gouji Toyokawa et al. All Copyright (c) 2014 are guarded by law and by SCIRP as a guardian.

\section{ABSTRACT}

Despite the aggressive pursuit of diagnostic and treatment modalities for lung cancer, the treatment outcomes are still not satisfactory, and even patients with surgically resectable non-small cell lung cancer (NSCLC) are often at considerable risk of suffering recurrence and/or death from lung cancer. Regarding the treatment of patients with locally advanced, resectable NSCLC, several retrospective and prospective studies have shown the significance of multimodality treatments with preoperative chemoradiotherapy and surgical treatment. However, no definitive treatment strategies for locally advanced NSCLC patients have yet been established. One of the reasons for the lack of established treatment strategies for patients with locally advanced NSCLC is considered to be the heterogeneity of the population, i.e., cT4N0, cT3-4N1 and cT1a-3N2 tumors are included in stage IIIA disease, and superior sulcus tumors (SSTs) are also included in this classification. With regard to SST, two representative prospective phase II trials indicated the efficacy of surgical treatment following concurrent radiation and chemotherapy. In a study conducted by the Southwest Oncology Group, 110 patients with superior sulcus NSCLC were treated with two cycles of cisplatin and etoposide concurrently with 45 gray (Gy) of radiation, followed by surgical treatment and two additional cycles of chemotherapy postoperatively. The response rate (RR) to the preoperative chemoradiotherapy was $86 \%$, and 83 patients $(76 \%)$ were able to undergo complete resection. A pathological complete response (CR) was observed in 61 patients (56\%), and the five-year survival of all patients and those undergoing complete resection was $44 \%$ and $54 \%$, respectively. A phase II study conducted by the Japan Clinical Oncology Group examined the safety and efficacy of preoperative concurrent chemoradiotherapy using mitomycin, vinblastin and cisplatin followed by surgical treatment. Seventy-six patients with SST were enrolled in this study, and all received chemotherapy using two cycles of MVP concurrently with 45 Gy of radiation, followed by surgery. Neoadjuvant chemoradiotherapy resulted in a $61 \% \mathrm{RR}$, and pathological complete resection was successfully achieved in 51 patients (68\%). A pathological CR was observed in 12 patients $(\mathbf{1 6 \%})$, and the disease-free and overall survival rates at five years were $45 \%$ and $56 \%$, respectively. Both studies showed the efficacy and tolerability of the multimodality treatment for SST, thus suggesting that multimodality treatment with preoperative chemoradiotherapy followed by surgery may therefore be an effective treatment for resectable SST. We herein review the results of retrospective and prospective studies while assessing the treatment outcomes of NSCLC patients with SST.

\section{KEYWORDS}

Locally Advanced Non-Small Cell Lung Cancer; Superior Sulcus Tumor; Multimodality Therapy

\section{Introduction}

Lung cancer is the leading cause of cancer death in many developed countries including Japan [1]. Despite the aggressive pursuit of both diagnostic and treatment modali- ties for lung cancer, the treatment outcomes for lung cancer still remain unsatisfactory, and even patients with surgically resectable non-small cell lung cancer (NSCLC) are at considerable risk of both recurrence and death from lung cancer. When treating patients with lung can- 
cer, it is therefore crucial to comprehensively understand the patient background, the exact stage of the tumor, the histological subtype and the molecular pathogenesis of the lung cancer. The recommended treatment strategies vary according to the stage of lung cancer. Complete resection of one lobe or more, in which the lesion is located, is recommended for patients with stage I-II disease [2-5], and some populations with these stages have been shown to benefit from adjuvant chemotherapy, including platinum-based chemotherapy or uracil and tegafur (also referred to as UFT) [6-8]. Patients with stage IV disease are mainly treated by chemotherapy [9], while palliative treatment, such as irradiation for metastasized bone or brain lesions is optionally administered. For this population, treatment strategies based on the mutation status of oncogenic drivers, such as the epidermal growth factor receptor (EGFR), anaplastic lymphoma kinase (ALK) and so on, are critical, because those harboring the mutations of these genes benefit from treatment with specific kinase inhibitors [10-12]. Regarding the treatment of patients with locally advanced, i.e., stage III, NSCLC, surgical treatment alone has been shown to be insufficient for the control of both local and distant disease [13-16]. Although several reports have indicated the efficacy and safety of concurrent chemotherapy and radiation followed by surgery, the treatment of patients with locally advanced NSCLC still remains controversial. Superior sulcus tumors (SSTs) are included in locally advanced NSCLC, and multimodality treatment with preoperative chemoradiotherapy followed by surgical resection is currently a standard treatment strategy for resectable SST.

We herein review the results of both retrospective and prospective studies analyzing the outcomes of the multimodality treatment for NSCLC patients with SST. Although the surgical techniques for SST are very challenging and several approaches have been developed [17-21], these technical aspects are not evaluated in this review article.

\section{Evidence for the Treatment of SST}

\subsection{Definition of SST as a Surgical Subject}

SSTs are bronchogenic cancers that typically produce unrelenting pain in the distribution of the eighth cervical and first and second thoracic nerve roots, and are also associated with Horner's syndrome and motor deficits of the intrinsic hand muscles (known as Pancoast's syndrome). The apical location of the tumor is associated with its tendency to cause severe pain, and functional deficits are caused by its locally invasive nature. Paulson et al. introduced an approach involving neoadjuvant radiotherapy followed by surgical resection, and this approach became the standard model from 1975 to 1997 [22]. Rusch et al. reviewed retrospective studies which analyzed the treatment outcomes for superior pulmonary cancer, and showed that the survival at five years ranges from $25 \%$ to $30 \%$ [23]. In studies other than that reported by Martínez-Monge et al. [24], radiotherapy alone was followed by surgery. The percentage of patients able to undergo a complete resection ranged from $48 \%$ to $76 \%$, and the combined treatment modality led to a two-year survival ranging from $34 \%$ to $64 \%$ and a five-year survival ranging from $17.4 \%$ to $56 \%$. Among the studies reviewed by Rusch et al., only the study conducted by Martínez-Monge et al. employed concurrent chemoradiotherapy (MVP: mitomycin, vinblastin and cisplatin) followed by surgical treatment; however, the survival benefit of this multidisciplinary therapy was not observed in comparison to other studies. As a matter of course, much care should be taken when interpreting these data, since the findings were obtained from retrospective studies that were carried out on a small number of patients. In addition, beginning in the late 1990s, several investigators conducted retrospective studies on the treatment for SST [23-32]. In addition, three prospective trials conducted by Marra et al., the Southwest Oncology Group (SWOG) and the Japan Clinical Oncology Group (JCOG) investigated the feasibility of surgery following neoadjuvant concurrent chemoradiotherapy for patients with SST [3336].

The question thus arises, are SSTs truly indicated for surgery? Although performing surgical resection has long been considered impossible due to the unique anatomical location of SST, the development of multimodality treatment and novel techniques, as mentioned above, thus makes this challenging subset of lung cancer amenable to potential curative treatment [37]. Several retrospective studies have demonstrated the safety and efficacy of extended surgical resection in cases of SST. For instance, according to a report by the M.D. Anderson Cancer Center, patients with SST invading the vertebrae were safely and effectively treated with a multidisciplinary surgical approach, including total vertebrectomy, partial vertebrectomy and either neural foramina or transverse process resection [26]. In two representative studies conducted by the SWOG and JCOG, SST patients with invasion to the chest wall or subclavian vessels (T3) and spine, aorta or superior vena cava (T4) were included in the analyses. According to the JCOG study, although hilar nodal (N1) and/or ipsilateral nodal (N3) involvement was judged to be eligible, SSTs with mediastinal nodal involvement (N2) were not included [36]; only hilar nodal metastasis was included in the SWOG study $[34,35]$. Combined resection of the invaded adjacent structures, as indicated above, was achieved without any 
severe surgery-related complications. Importantly, multimodality treatment with concurrent chemoradiotherapy followed by surgery achieved better outcomes than those observed in the retrospective studies. Based on these findings, SST lesions invading the surrounding vital structures and/or with metastasis to the hilar or supraclavicular lymph nodes are thus considered to be indicated for surgery.

\subsection{Results of Retrospective Studies}

Table 1 shows the clinical outcomes of the seven representative retrospective trials performed since the early 1990s. According to these studies, although the clinicopathological characteristics of the enrolled population, such as the number, stage and treatment modalities were heterogeneous, the rate of complete resection and fiveyear survival ranged from $23 \%$ to $100 \%$, and from $0 \%$ to $65 \%$, respectively, which were similar to those reported in the review by Rusch et al. Additionally, the surgical mortality rate was $0 \%-8.9 \%$. According to a report by Hagan et al. [25], unresected primary disease, a low performance status, T4 stage and positive node status were all associated with a significantly poorer prognosis, whereas Komaki et al. [27] showed that the overall predictors of the five-year survival were weight loss, supraclavicular fossa or vertebral body involvement, the stage of the disease and the completion of surgical treatment. Multivariate analyses showed a significant impact of the $\mathrm{T}$ and $\mathrm{N}$ status and complete resection on survival [23]. In contrast, another study showed that the surgical approach, postoperative radiotherapy and postoperative chemotherapy did not improve the five-year survival, while preoperative radiotherapy did [28]. Furthermore, Alifano et al. showed that the completeness of resection and the absence of any associated major comorbidities had an independent positive prognostic value [29].

Regarding recently published reports [30-32], Pourel et al. described the results of concurrent cisplatin (CDDP)/etoposide (VP-16) with 3D-conformal radiotherapy (45 Gy/25 Fr.) followed by surgical resection for patients with SST [30]. Among the 107 patients included in their study, a complete resection was performed in 71 patients, and five patients (6.9\%) died during the first three months postoperatively. The three-year overall survival (OS) rate was $40 \%$, and the patients undergoing complete resection achieved a significantly higher threeyear OS rate of $51 \%$ than did the patients who did not receive surgical resection. In a report by Kappers and colleagues, 39 patients with SST received combined modality treatment including surgery, and 25 patients were treated with accelerated radiation (66 Gy/24 Fr.) with concurrent daily CDDP [31]. Among the 25 patients who underwent chemoradiotherapy, 22 patients were able to undergo surgery. All resections were complete without any local recurrences, and the resected patients achieved a significantly better OS than the unresected patients. In addition, our institution reported the evolution of the treatment outcomes with multimodality treatment for NSCLC of the superior sulcus [32]. Fifty-seven patients with STS who were treated between 1982 and 2007 were retrospectively analyzed, and 47\% of the patients underwent preoperative chemoradiation followed by surgery, and 53\% underwent other treatments, such as surgery alone, surgery plus radiotherapy or chemoradiation. The five-year survival rates increased significantly from $42.6 \%$ (1982-1994) to 65.4\% (19952007). Intriguingly, the five-year survival of patients who received neoadjuvant chemoradiotherapy followed by surgery $(\mathrm{n}=27)$ was better than that of those who received another treatment regimen with surgery $(\mathrm{n}=22$, $64.6 \%$ versus $49.6 \%$; $\mathrm{p}=0.044)$. The complete resection rate was similar to that in other reports, and the five-year survival in patients with a complete resection after chemoradiotherapy was $70.4 \%$. Although these were retrospective studies which analyzed only a small number of patients with SST, they nevertheless showed that multimodality treatment with preoperative chemoradiation followed by surgical resection can achieve good treatment outcomes.

\subsection{Results of Prospective Studies}

Only three prospective studies have so far examined the feasibility of neoadjuvant chemoradiotherapy followed by surgery for patients with SST (Table 2). Marra and associates conducted a prospective feasibility study to determine whether multimodality treatment improves the local control and survival [33]. Thirty-one patients with SST (T3: 81\%; T4: 19\%) were enrolled in the study, and all of the patients could receive the induction chemotherapy with three courses of split-dose CDDP and VP16 or paclitaxel, followed by concurrent chemoradiotherapy with a course of CDDP/VP-16 with concurrent 45 Gy hyperfractionated accelerated radiation. Twentynine (94\%) patients were eligible for surgical resection, and a complete resection was achieved in 94\% of these patients, with a thirty-day mortality rate of $6.4 \%$. Fortyeight percent of the patients achieved a partial response with induction treatment, and a pathological complete response (CR) was observed in 13 (4.9\%) patients. The actual OS at five years for the entire population was $46 \%$, with a median survival of 54 months. The five-year OS rates were $63 \%$ for the complete responders versus 35\% for the partial responders $(p=0.10)$; patients receiving paclitaxel-based induction therapy had a higher five-year OS rate compared with the patients treated with VP-16 (76\% versus $31 \%$; $p=0.16$ ). Recurrences were observed 

the Lung: A Review Article

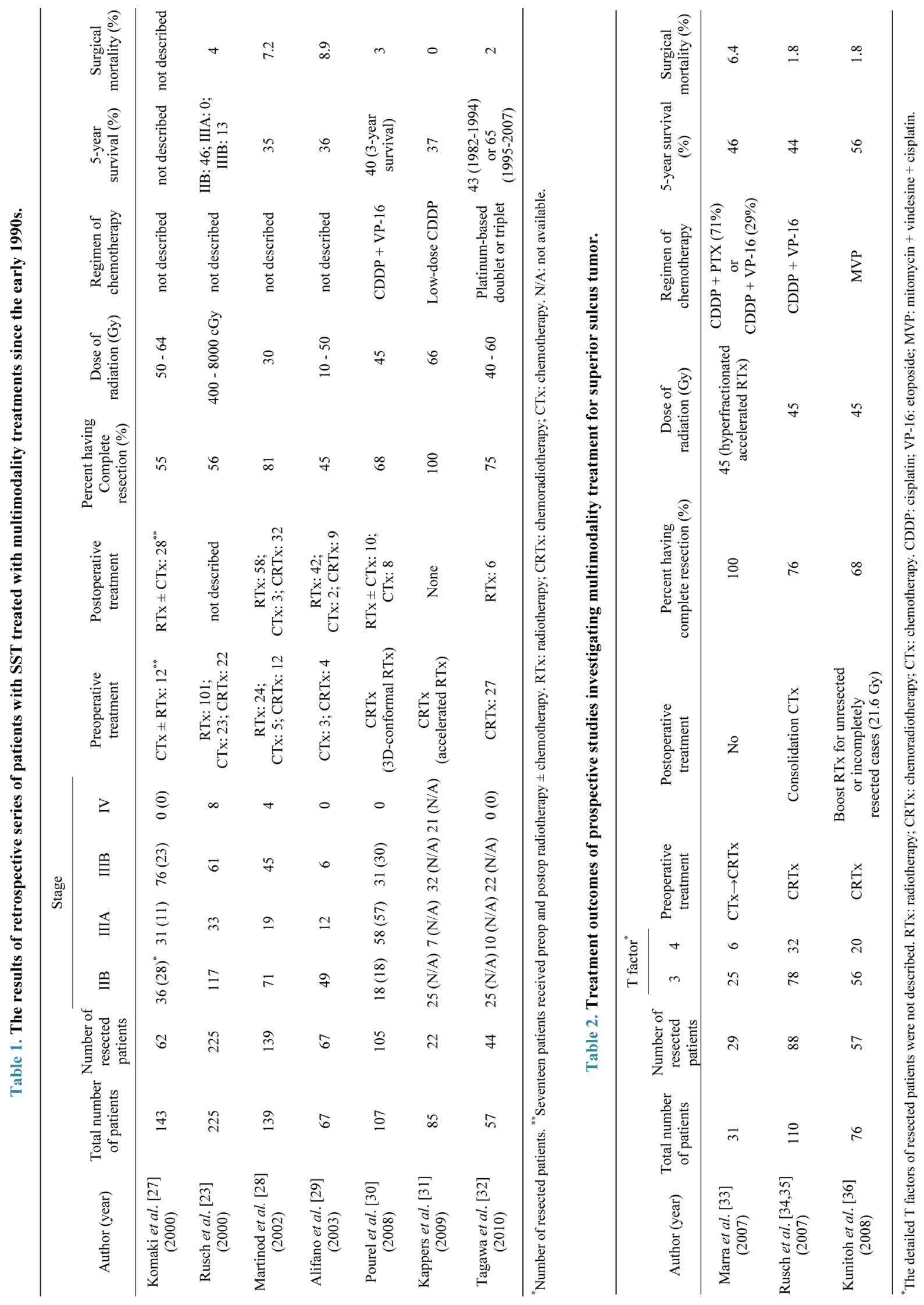


as 11 events in nine (29\%) patients: one (3.2\%) patient suffered a local recurrence, seven (22.6\%) patients suffered distant metastases (the most common site was the brain: $12.9 \%$ ) and one (3.2\%) patient suffered both at the same time.

A prospective phase II study was conducted by the SWOG to evaluate the safety and efficacy of preoperative chemoradiotherapy followed by surgical resection in patients with superior sulcus NSCLC [34,35]. One hundred and ten patients with T3-4, N0-1 SST were enrolled in SWOG 9416 (Intergroup Trial 0160) from April 1995 to November 1999, and they were treated with two cycles of CDDP and VP-16 concurrently with $45 \mathrm{~Gy}$ of radiation. Eighty-eight patients (80\%) could undergo surgical resection and two additional cycles of chemotherapy postoperatively. The response rate (RR) to the preoperative chemoradiotherapy was $86 \%$, and 83 patients (76\%) had a complete resection. A pathological CR was observed in 61 patients (56\%), and the five-year survival of all patients and those undergoing complete resection were $44 \%$ and $54 \%$, respectively. Although the $\mathrm{T}$ stage had no impact on the survival, a pathological CR was associated with a prolonged survival compared with those without a pathological CR. Additionally, induction therapy was shown to be well tolerated, and postoperative pulmonary complications were the most common complication, with pneumonia occurring in $13.6 \%$ of patients, and postoperative death occurred in two patients (2.3\%) due to multisystem failure. Although local recurrence developed in only 10 patients, disease progression occurred mainly at distant sites, and the most common single site was the brain, with recurrence in this site in 19 of 57 patients.

The JCOG prospectively examined the safety and efficacy of preoperative concurrent chemoradiotherapy using MVP followed by surgical treatment [36]. From 1999 to 2002, 76 patients with SST were enrolled in the JCOG 9806 trial, and they received chemotherapy with two cycles of MVP concurrently with 45 Gy of radiation, followed by surgery. Fifty and 20 patients had T3 and T4 disease, respectively, and fifty-seven patients (76\%) underwent surgical resection, with a pathological complete resection achieved in 51 patients (68\%). Neoadjuvant chemoradiotherapy resulted in a $61 \% \mathrm{RR}$, and a pathological CR was observed in 12 patients. The disease-free and OS rates at five years were $45 \%$ and $56 \%$, respectively. The clinical $\mathrm{T}$ stage was proven to be a prognostic factor, and patients with clinical T3 disease had a better outcome than those with clinical $\mathrm{T} 4$ disease, i.e., the survival rates at five years were $61 \%$ versus $40 \%$. Consistent with the results of the SWOG 9416, the survival of the 12 patients with a pathological CR was especially favorable compared with that of the patients who did not experience a pathological CR. Although more than $80 \%$ of the patients experienced neutropenia/leukopenia, chemoradiotherapy was reported to be generally well tolerated. The major postoperative morbidities included adult respiratory distress syndrome (ARDS) in two patients, empyema in two patients, chylothorax in two patients, and pneumonitis in two patients, and the total number of toxic deaths was three, including one caused by septic shock during the induction, one by delayed postoperative bleeding, and one by the development of ARDS after off-protocol, salvage surgery. For completely resected cases, relapse at distant sites was the most frequent relapse pattern, while locoregional relapse was predominant in unresected or incompletely resected cases.

One possible concern, as observed in both the JCOG and SWOG studies, is the management of patients with metastasis to the mediastinal nodes (N2). As indicated above, the JCOG study excluded patients with N2 disease, and N2 regions (as well as N1 regions, even if involved) were not included in the irradiation field. Since the data obtained by the JCOG showed an increase in toxicity associated with the inclusion of $\mathrm{N} 1$ and $\mathrm{N} 2$ disease in the irradiation field [38] and clinical N2 disease has been shown to be the poorest prognostic factor [23], these exclusion criteria appear to be reasonable. Furthermore, to not irradiate N2 disease is considered to be beneficial when conducting surgical resection of both the upper lobe in which the tumor is located and the invaded adjacent structures, such as vertebrae. Therefore, when treating SSTs associated with clinical N2 disease, obtaining a pretreatment diagnosis is essential. In addition, the recent development of less invasive diagnostic methods, such as endobronchial ultrasound-guided transbronchial needle aspiration, is considered to improve the diagnostic process [39].

Although the regimens of chemotherapy used in the two representative prospective studies were not thirdgeneration [34-36], the five-year survival rates of the two prospective trials were superior to those of historical controls, i.e., $44 \%$ and $56 \%$. Based on these findings, surgical treatment following concurrent chemoradiotherapy is considered to be a standard treatment for patients with resectable superior sulcus NSCLC.

With regard to postoperative chemoradiotherapy, there was one prospective phase II trial conducted by Gomez et al. [40]. From 1994 to 2010, 32 patients with SST were enrolled in the study, and they were treated with surgical treatment followed by concurrent chemoradiotherapy using CDDP + VP-16 (60 Gy if the surgical margins were negative; $64.8 \mathrm{~Gy}$ if the margins were positive). Complete resection was possible in 23 patients (72\%), and the surgical mortality was $0 \%$. Although the 
five-year disease-free survival and OS rates were 45\% and $50 \%$, respectively, which were comparable to those of the SWOG and JCOG studies, there were some critical problems in that study: the criteria for surgical treatment were unclear, the inclusion period was too long and the number of cases enrolled in the study was small. Therefore, for the present, adjuvant concurrent chemoradiotherapy after surgical treatment is not recommended for patients with SST.

\section{Future Challenges}

Although the treatment outcomes reported by the SWOG and JCOG were sufficiently successful to recommend concurrent chemoradiation followed by surgical resection [34-36], it is likely that there is still room for improvement in treatment outcomes for SST. One of factors for such possible improvement is assumed to be the identification of which chemotherapeutic regimen should be combined with radiotherapy. Although the chemotherapeutic drugs used in studies by the SWOG and JCOG included radiosensitizing drugs, they were not thirdgeneration chemotherapeutic agents. Apart from SST, a phase III study was performed by the West Japan Thoracic Oncology Group to compare the second- and thirdgeneration chemotherapeutic regimens concurrently used with radiotherapy (60 Gy) in patients with unresectable stage III NSCLC [41]. Although no significant differences in the OS were observed between second- (mitomycin/vindesine/CDDP) and third-generation (irinotecan/carboplatin or paclitaxel/carboplatin) regimens, the third-generation regimens with paclitaxel/carboplatin achieved a more favorable toxicity profile in comparison to other regimens, thus leading to the conclusion that the regimen with paclitaxel/carboplatin should be considered as the standard regimen for the treatment of locally advanced unresectable NSCLC. Furthermore, Ichinose et al. reported excellent outcomes for a prospective phase II study that examined the efficacy and safety of the treatment with S-1 plus CDDP with concurrent radiotherapy (60 Gy) for patients with locally advanced NSCLC [42]: an objective response rate was achieved in $84 \%$ of the 50 evaluable patients; the one- and two-year OS rates were $88 \%$ and $70 \%$, respectively, and the median PFS was 20 months, which was better than that reported by Yamamoto et al. (paclitaxel/carboplatin: 9.5 months) [41]; the toxicity profile was acceptable. The efficacy and safety of the treatment using S-1/CDDP with concurrent radiotherapy was also confirmed for resectable patients with stage III NSCLC [43]. Based on these data, the regimen of S-1/CDDP used concurrently with radiation might also be also promising for patients with SST, and should be prospectively investigated for its safety and efficacy.

Another point for possible improvement is considered to be the dose of radiation. Although many studies adopted less than 50 Gy of radiation as shown in Tables 1 and 2, some studies treated SST patients with radical doses of radiation. For example, according to the report by Kappers et al., 39 patients with SST were treated with concurrent chemoradiotherapy, and the chemoradiotherapy was composed of accelerated radiation (66 Gy in fractions of $2.75 \mathrm{~Gy}$, which is definitive dose radiation) with daily CDDP. Among the 39 patients, 22 patients were eligible for surgical resection, all of whom achieved complete resection. Importantly, no fatal toxicity or treatment-related death was observed. Although a pathological CR was more frequently seen in the study (13/22: $62 \%)$, no survival benefit was observed. Furthermore, there exist some studies indicating the survival benefit of high-dose radiation over standard-dose radiation without any increase of mortality and morbidity in resected stage III patient with NSCLC [44], and the safety of induction hyperfractionated radiation (69.6 Gy) and concurrent chemotherapy followed by surgery in stage III NSCLCs was shown by a phase I/II study [45]. In contrast, a randomized phase III study comparing the OS of patients treated with standard-dose (SD) (60 Gy) or high-dose (HD) (74 Gy) radiation with concurrent chemotherapy failed to show the superiority of HD radiation over SD radiation in patients with stage III NSCLC (median survival time: $\mathrm{HD}$ versus $\mathrm{SD}, 19.5$ versus 28.7 months, $\mathrm{p}=$ 0.0007 ) [46]. In view of these results, it is hard to say that HD radiation is superior to SD radiation, and the appropriate dose of radiation concurrently administered with chemotherapy in patients with locally advanced NSCLC such as SST or stage III should be investigated by future prospective studies.

Another concern to be solved is a high rate of distant relapse after surgical resection. The distant relapse rates, especially recurrence in the brain, are higher than the locoregional relapse rates. With regard to the control of brain metastases after multimodality treatment for locally advanced NSCLC (not SST), a phase III prospective study was conducted to compare prophylactic cranial irradiation (PCI) versus observation after the multimodality treatment [47]. PCI decreased the incidence of brain metastasis; however, neither the OS nor DFS improved. The authors concluded that PCI is not recommended, since there was no evidence of a survival benefit in patients with locally advanced NSCLC. The fact that the control of brain metastasis failed to improve the patient survival led to the idea to examine the use of intensive neoadjuvant and/or adjuvant chemotherapy after surgical resection. The administration of molecular targeted therapy, such as EGFR-TKI and ALK inhibitors [10-12], for patients with SST in the neoadjuvant and/or adjuvant setting might be promising, which should be 
clarified by prospective studies, although the molecular and genetic profiling of SST has yet to be performed. For instance, Okamoto et al. conducted a feasibility study to examine the safety and toxicity profile of daily gefitinib treatment concurrently administered with thoracic radiation in patients with unresectable, locally advanced NSCLC [48]. Although the study was closed because of the lower rate of treatment completion than expected before the study initiation, and only two patients harbored sensitive EGFR mutations, both of these two patients completed the designed treatment schedule and exhibited an OS greater than five years. It is difficult to conclude whether concurrent gefitinib treatment with radiotherapy is safe and effective for locally advanced NSCLC based on these two cases; however, this therapeutic strategy might hold promise. These results might therefore be applicable for patients with SST, which is a very small subset of NSCLC.

\section{Conclusion}

We herein reviewed both representative retrospective and prospective studies of the treatment modalities, especially induction therapy followed by surgery, for patients with SST. At present, induction treatment with preoperative chemoradiation followed by surgery, appears to be a potent treatment option for patients with resectable SST, and future studies are warranted to improve the survival rates, as well as the control of both local and distant relapse, especially in the brain.

\section{Acknowledgements}

None of the authors have any conflicts of interest to declare. We thank Brian Quinn, Editor-in-Chief, Japan Medical Communication, for providing critical comments on the manuscript.

\section{REFERENCES}

[1] A. J. Alberg, et al., "American College of Chest Physicians. Epidemiology of Lung Cancer: ACCP EvidenceBased Clinical Practice Guidelines (2nd Edition),” Chest, Vol. 132, No. 3, 2007, pp. 29S-55S.

[2] R. J. Ginsberg, et al., "Randomized Trial of Lobectomy versus Limited Resection for T1 N0 Non-Small Cell Lung Cancer. Lung Cancer Study Group,” Annals of Thoracic Surgery, Vol. 60, No. 3, 1995, pp. 615-622. http://dx.doi.org/10.1016/0003-4975(95)00537-U

[3] H. Asamura, et al., "A Japanese Lung Cancer Registry Study: Prognosis of 13,010 Resected Lung Cancers," Journal of Thoracic Oncology, Vol. 3, No. 1, 2008, pp. 46-52. http://dx.doi.org/10.1097/JTO.0b013e31815e8577

[4] T. Goya, et al., "Prognosis of 6644 Resected Non-Small Cell Lung Cancers in Japan: A Japanese Lung Cancer Registry Study,” Lung Cancer, Vol. 50, No. 2, 2005, pp.
227-234. http://dx.doi.org/10.1016/j.lungcan.2005.05.021

[5] N. Sawabata, et al., "Japanese Lung Cancer Registry Study of 11,663 Surgical Cases in 2004: Demographic and Prognosis Changes over Decade," Journal of Thoracic Oncology, Vol. 6, No. 7, 2011, pp. 1229-1235. http://dx.doi.org/10.1097/JTO.0b013e318219aae2

[6] H. Kato, et al., "A Randomized Trial of Adjuvant Chemotherapy with Uracil-Tegafur for Adenocarcinoma of the Lung," New England Journal of Medicine, Vol. 350, No. 17, 2004, pp. 1713-1721. http://dx.doi.org/10.1056/NEJMoa032792

[7] J. P. Pignon, et al., "Lung Adjuvant Cisplatin Evaluation: A Pooled Analysis by the LACE Collaborative Group," Journal of Clinical Oncology, Vol. 26, No. 21, 2008, pp. 3552-3559. http://dx.doi.org/10.1200/JCO.2007.13.9030

[8] S. Heon, et al., "Adjuvant Chemotherapy for Surgically Resected Non-Small Cell Lung Cancer,” Journal of Thoracic Cardiovascular Surgery, Vol. 144, No. 3, 2012, pp. S39-S42. http://dx.doi.org/10.1016/j.jtcvs.2012.03.039

[9] Non-Small Cell Lung Cancer Collaborative Group, “Chemotherapy and Supportive Care versus Supportive Care Alone for Advanced Non-Small Cell Lung Cancer," Cochrane Database of Systematic Reviews, Vol. 12, No. 5, 2010, Article ID: CD007309.

[10] T. Mitsudomi, et al., "Gefitinib versus Cisplatin plus Docetaxel in Patients with Non-Small-Cell Lung Cancer Harbouring Mutations of the Epidermal Growth Factor Receptor (WJTOG3405): An Open Label, Randomised Phase 3 Trial,” Lancet Oncology, Vol. 11, No. 2, 2010, pp. 121-128. http://dx.doi.org/10.1016/S1470-2045(09)70364-X

[11] A. T. Shaw, et al., "Crizotinib versus Chemotherapy in Advanced ALK-Positive Lung Cancer,” New England Journal of Medicine, Vol. 368, No. 25, 2013, pp. 23852394. http://dx.doi.org/10.1056/NEJMoa1214886

[12] T. Seto, et al., "CH5424802 (RO5424802) for Patients with ALK-Rearranged Advanced Non-Small-Cell Lung Cancer (AF-001JP Study): A Single-Arm, Open-Label, Phase 1-2 Study," Lancet Oncology, Vol. 14, No. 7, 2013, pp. 590-598.

http://dx.doi.org/10.1016/S1470-2045(13)70142-6

[13] F. G. Pearson, et al., "Significance of Positive Superior Mediastinal Nodes Identified at Mediastinoscopy in Patients with Resectable Cancer of the Lung," Journal of Thoracic and Cardiovascular Surgery, Vol. 83, No. 1, 1982, pp. 1-11.

[14] N. Martini, et al., "The Role of Surgery in N2 Lung Cancer,” Surgical Clinics of North America, Vol. 67, No. 5, 1987, pp. 1037-1049.

[15] Y. Watanabe, et al., "Aggressive Surgical Intervention in N2 Non-Small Cell Cancer of the Lung," Annals of Thoracic Surgery, Vol. 51, No. 2, 1991, pp. 253-261. http://dx.doi.org/10.1016/0003-4975(91)90797-T

[16] T. Funatsu, et al., "The Role of Mediastinoscopic Biopsy in Preoperative Assessment of Lung Cancer," Journal of Thoracic and Cardiovascular Surgery, Vol. 104, No. 6, 1992, pp. 1688-1695.

[17] R. R. Shaw, et al., “Treatment of Superior Sulcus Tumor 
by Irradiation Followed by Resection,” Annals of Surgery, Vol. 154, No. 1, 1961, pp. 29-40. http://dx.doi.org/10.1097/00000658-196107000-00003

[18] A. Masaoka, et al., "Anterior Approach for Tumor of the Superior Sulcus," Journal of Thoracic and Cardiovascular Surgery, Vol. 78, No. 3, 1979, pp. 413-415.

[19] H. Niwa, et al., "Surgical Therapy for Apical Invasive Lung Cancer: Different Approaches According to Tumor Location,” Lung Cancer, Vol. 10, No. 1-2, 1993, pp. 6371. http://dx.doi.org/10.1016/0169-5002(93)90310-T

[20] P. G. Dartevelle, et al., "Anterior Transcervical-Thoracic Approach for Radical Resection of Lung Tumors Invading the Thoracic Inlet," Journal of Thoracic and Cardiovascular Surgery, Vol. 105, No. 6, 1993, pp. 1025-1034.

[21] D. Grunenwald, et al., "Transmanubrial Osteomuscular Sparing Approach for Apical Chest Tumors,” Annals of Thoracic Surgery, Vol. 63, No. 2, 1997, pp. 563-566. http://dx.doi.org/10.1016/S0003-4975(96)01023-5

[22] D. L. Paulson, "Carcinomas in the Superior Pulmonary Sulcus,” Journal of Thoracic and Cardiovascular Surgery, Vol. 70, No. 6, 1975, pp. 1095-1104.

[23] V. W. Rusch, et al., "Factors Determining Outcome after Surgical Resection of T3 and T4 Lung Cancers of the Superior Sulcus," Journal of Thoracic and Cardiovascular Surgery, Vol. 119, No. 6, 2000, pp. 1147-1153. http://dx.doi.org/10.1067/mtc.2000.106089

[24] R. Martínez-Monge, et al., "Combined Treatment in Superior Sulcus Tumors," American Journal of Clinical Oncology, Vol. 17, No. 4, 1994, pp. 317-322. http://dx.doi.org/10.1097/00000421-199408000-00007

[25] M. P. Hagan, et al., "Superior Sulcus Lung Tumors: Impact of Local Control on Survival,” Journal of Thoracic and Cardiovascular Surgery, Vol. 117, No. 6, 1999, pp. 1086-1094. http://dx.doi.org/10.1016/S0022-5223(99)70244-8

[26] S. Gandhi, et al., "A Multidisciplinary Surgical Approach to Superior Sulcus Tumors with Vertebral Invasion,” Annals of Thoracic Surgery, Vol. 68, No. 5, 1999, pp. 17781784. http://dx.doi.org/10.1016/S0003-4975(99)01068-1

[27] R. Komaki, et al., "Outcome Predictors for 143 Patients with Superior Sulcus Tumors Treated by Multidisciplinary Approach at the University of Texas M.D. Anderson Cancer Center," International Journal of Radiation Oncology, Biology, Physics, Vol. 48, No. 2, 2000, pp. 347354. http://dx.doi.org/10.1016/S0360-3016(00)00736-7

[28] E. Martinod, et al., "Management of Superior Sulcus Tumors: Experience with 139 Cases Treated by Surgical Resection,” Annals of Thoracic Surgery, Vol. 73, No. 5, 2002, pp. 1534-1540. http://dx.doi.org/10.1016/S0003-4975(02)03447-1

[29] M. Alifano, et al., "Surgical Treatment of Superior Sulcus Tumors: Results and Prognostic Factors,” Chest, Vol. 124, No. 3, 2003, pp. 996-1003. http://dx.doi.org/10.1378/chest.124.3.996

[30] N. Pourel, et al., “Concurrent Cisplatin/Etoposide plus 3D-Conformal Radiotherapy Followed by Surgery for IIB (Superior Sulcus T3N0)/III Non-Small Cell Lung Cancer
Yields a High Rate of Pathological Complete Response,” European Journal of Cardiothoracic Surgery, Vol. 33, No. 5, 2008, pp. 829-836.

http://dx.doi.org/10.1016/j.ejcts.2008.01.063

[31] I. Kappers, et al., "Results of Combined Modality Treatment in Patients with Non-Small-Cell Lung Cancer of the Superior Sulcus and the Rationale for Surgical Resection,” European Journal of Cardiothoracic Surgery, Vol. 36, No. 4, 2009, pp. 741-746. http://dx.doi.org/10.1016/j.ejcts.2009.04.069

[32] T. Tagawa, et al., "Non-Small Cell Lung Carcinoma of the Superior Sulcus: The Evolution of Treatment Outcomes with Multimodality Treatment at a Single Institution,” Journal of Surgical Oncology, Vol. 101, No. 6, 2010, pp. 495-499.

[33] A. Marra, et al., "Induction Chemotherapy, Concurrent Chemoradiation and Surgery for Pancoast Tumour," European Respiratory Journal, Vol. 29, No. 1, 2007, pp. 117-126. http://dx.doi.org/10.1183/09031936.00108205

[34] V. W. Rusch, et al., "Induction Chemoradiation and Surgical Resection for Non-Small Cell Lung Carcinomas of the Superior Sulcus: Initial Results of Southwest Oncology Group Trial 9416 (Intergroup Trial 0160),” Journal of Thoracic and Cardiovascular Surgery, Vol. 121, No. 3, 2001, pp. 472-483. http://dx.doi.org/10.1067/mtc.2001.112465

[35] V. W. Rusch, et al., "Induction Chemoradiation and Surgical Resection for Superior Sulcus Non-Small-Cell Lung Carcinomas: Long-Term Results of Southwest Oncology Group Trial 9416 (Intergroup Trial 0160),” Journal of Clinical Oncology, Vol. 25, No. 3, 2007, pp. 313-318. http://dx.doi.org/10.1200/JCO.2006.08.2826

[36] H. Kunitoh, et al., "Phase II Trial of Preoperative Chemoradiotherapy Followed by Surgical Resection in Patients with Superior Sulcus Non-Small-Cell Lung Cancers: Report of Japan Clinical Oncology Group Trial 9806,” Journal of Clinical Oncology, Vol. 26, No. 4, 2008, pp. 644-649. http://dx.doi.org/10.1200/JCO.2007.14.1911

[37] V. W. Rusch, "Management of Pancoast Tumours," Lancet Oncology, Vol. 7, No. 12, 2006, pp. 997-1005. http://dx.doi.org/10.1016/S1470-2045(06)70974-3

[38] H. Kunitoh, et al., "A Pilot Trial of Preoperative MVPCombined Chemoradiotherapy: Mitomycin C (MMC), Vindesine (VDS), and Cisplatin (CDDP), Concurrently Given with Thoracic Radiotherapy (TRT) in N2 NonSmall Cell Lung Cancer (NSCLC)," Proceedings of American Society of Clinical Oncology, Vol. 19, 2000, p. 530a.

[39] K. G. Tournoy, et al., "Mediastinal Staging of Lung Cancer: Novel Concepts," Lancet Oncology, Vol. 13, No. 5, 2012, pp. e221-e229. http://dx.doi.org/10.1016/S1470-2045(11)70407-7

[40] D. R. Gomez, et al., "A Prospective Phase 2 Study of Surgery Followed by Chemotherapy and Radiation for Superior Sulcus Tumors,” Cancer, Vol. 118, No. 2, 2012, pp. 444-451. http://dx.doi.org/10.1002/cncr.26277

[41] N. Yamamoto, et al., "Phase III Study Comparing Second- and Third-Generation Regimens with Concurrent Thoracic Radiotherapy in Patients with Unresectable 
Stage III Non-Small-Cell Lung Cancer: West Japan Thoracic Oncology Group WJTOG0105,” Journal of Clinical Oncology, Vol. 28, No. 23, 2007, pp. 3739-3745. http://dx.doi.org/10.1200/JCO.2009.24.5050

[42] Y. Ichinose, et al., "S-1 plus Cisplatin with Concurrent Radiotherapy for Locally Advanced Non-Small Cell Lung Cancer: A Multi-Institutional Phase II Trial (West Japan Thoracic Oncology Group 3706)," Journal of Thoracic Oncology, Vol. 6, No. 12, 2011, pp. 2069-2075. http://dx.doi.org/10.1097/JTO.0b013e3182307e5a

[43] M. Yamaguchi, et al., "Preoperative Concurrent Chemoradiotherapy of S-1/Cisplatin for Stage III Non-Small Cell Lung Cancer,” Annals of Thoracic Surgery, Vol. 96, No. 5, 2013, pp. 1783-1789. http://dx.doi.org/10.1016/j.athoracsur.2013.06.036

[44] R. J. Cerfolio, et al., "Pulmonary Resection after HighDose and Low-Dose Chest Irradiation," Annals of Thoracic Surgery, Vol. 80, No. 4, 2005, pp. 1224-1230. http://dx.doi.org/10.1016/j.athoracsur.2005.02.091

[45] M. J. Edelman, et al., "Phase I/II Trial of Hyperfractionated Radiation and Chemotherapy Followed by Surgery in Stage III Lung Cancer,” Annals of Thoracic Surgery, Vol. 86, No. 3, 2008, pp. 903-910. http://dx.doi.org/10.1016/j.athoracsur.2008.06.022

[46] J. D. Bradley, et al., "A Randomized Phase III Comparison of Standard-Dose (60 Gy) versus High-Dose (74 Gy) Conformal Chemoradiotherapy with or without Cetuximab for Stage III Non-Small Cell Lung Cancer: Results on Radiation Dose in RTOG0617," Journal of Clinical Oncology, Vol. 31, 2013, Abstract 7501.

[47] E. M. Gore, et al., "Phase III Comparison of Prophylactic Cranial Irradiation versus Observation in Patients with Locally Advanced Non-Small-Cell Lung Cancer: Primary Analysis of Radiation Therapy Oncology Study RTOG 0214,” Journal of Clinical Oncology, Vol. 29, No. 3, 2011, pp. 272-278.

http://dx.doi.org/10.1200/JCO.2010.29.1609

[48] I. Okamoto, et al., "Single-Agent Gefitinib with Concurrent Radiotherapy for Locally Advanced Mutations of the Epidermal Growth Factor Receptor,” Lung Cancer, Vol. 72, No. 2, 2011, pp. 199-204. http://dx.doi.org/10.1016/j.lungcan.2010.08.016 